\title{
MODELAGEM E SIMULAÇÃO DE UM TERMOSSIFÃO VERTICAL
}

\author{
M. P. SANTOS, E. R. A. LIMA, A. L. H. COSTA \\ Universidade do Estado do Rio de Janeiro, Programa de Pós-Graduação em Engenharia Química \\ E-mail para contato: mischellepaiva@gmail.com
}

\begin{abstract}
RESUMO - Destilação é uma das técnicas de separação mais utilizadas no âmbito da indústria de processos químicos. Um elemento fundamental na operação de uma coluna de destilação corresponde ao refervedor, equipamento responsável por fornecer energia para o fracionamento. Em função desta condição, este trabalho apresenta a modelagem e simulação de um sistema completo de vaporização do tipo termossifão contendo todo o circuito, desde a saída da torre de destilação até o retorno da corrente bifásica. $\mathrm{O}$ modelo proposto corresponde a um sistema de equações algébrico-diferenciais contemplando balanços de massa, energia e momento. A aplicação do algoritmo proposto é ilustrada através de um exemplo referente à vaporização de uma substância pura, sendo possível descrever os perfis de distribuição de pressão, temperatura e vazão de líquido e vapor ao longo do circuito.
\end{abstract}

\section{INTRODUÇÃO}

A destilação possibilita a obtenção de produtos mais ricos em determinados componentes, por meio da interconexão de estágios de equilíbrio. Nesses estágios, uma corrente de vapor cruza com uma corrente de líquido, trocando calor e massa. $\mathrm{O}$ vapor ao percorrer o equipamento se enriquece dos componentes mais voláteis, enquanto o líquido dos componentes mais pesados (Caldas et al., 2007). Esses sistemas necessitam de trocadores de calor, com o objetivo de fornecer calor ao fundo da coluna, refervedores, e para remover calor do topo da coluna, condensadores (Indio do Brasil, 2004).

Uma alternativa de refervedor muito utilizada na prática corresponde ao termossifão vertical. Neste equipamento, a circulação da corrente de processo ocorre em função da diferença de densidade entre a coluna de líquido proveniente da torre e a mistura bifásica na saída do trocador de calor. Neste tipo de equipamento o mais comum é que a vaporização ocorra no interior dos tubos.

Esse tipo de trocador permite alcançar maiores valores de coeficiente de convecção, quando comparada a um kettle, entretanto, seu desempenho é bastante dependente das condições fluidodinâmicas. Por esta razão, a avaliação do desempenho destes equipamentos deve ser feita de forma criteriosa.

Em função deste cenário, este trabalho apresenta um modelo capaz de simular o comportamento termofluidodinâmico de refervedores verticais. Como resultado da simulação, é possível determinar a taxa de transferência de calor e a vazão de fluido em recirculação, aspectos fundamentais no desempenho do equipamento. 


\section{MODELAGEM}

As equações foram deduzidas e implementadas no integrador de forma adimensional, com objetivo de dar um significado às variáveis, simplificar, facilitar a implementação dos métodos numéricos e introduzir grupamentos adimensionais relevantes (Lage e Pinto, 2001).

O modelo para trocador de calor é composto por dois balanços de energia, um para o fluido quente e um para o fluido frio, complementado pela equação da taxa de transferência de calor.

O balanço de energia diferencial para a corrente pode ser representado por (Incropera et al., 2008):

$d H+m(v d v+g d z)=d Q-d W$

Para o escoamento ao longo do trocador de calor, não há trabalho mecânico e as variações de energia potencial e cinética podem ser desprezadas, quando comparadas com a variação na entalpia. Assim, a Equação 1 pode ser escrita para as seguintes formas particulares, para troca de calor sensível e latente, respectivamente:

$m C p d T=d Q=d H$

$m \lambda d x_{g}=d Q=d H$

A parcela referente à taxa de transferência de calor pode ser calculada pelo conceito de coeficiente global de transferência de calor:

$d Q=U d A\left(T_{H}-T\right)$

Sabendo que:

$d A=N t t \pi D \mathrm{dL}$

Substituindo a Equação 5 nas Equações 3 e 4, tem-se:

$\frac{d H}{d L}=N t t \pi D U\left(T_{H}-T\right)$

O Coeficiente global de transferência de calor $(U)$ foi calculado pela seguinte equação:

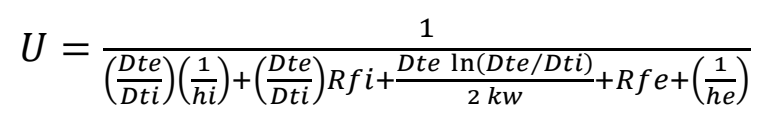

sendo he o coeficiente de transferência de calor externo, para o qual adotou-se o valor de $8500 \mathrm{~W} / \mathrm{m}^{2}$ K. Já o coeficiente de transferência de calor interno, $h i$ foi calculado pela abordagem de Chen, de acordo com Smith (1986), por meio de duas contribuições: ebulição convectiva e ebulição nucleada. A contribuição da convecção forçada foi calculada pelo produto entre o coeficiente de convecção forçada no líquido e um fator de amplificação, função do parâmetro de Martinelli. Essa contribuição está associada à intensificação da convecção forçada pela mudança de fase. Já a contribuição da ebulição nucleada está diretamente ligada à formação de bolhas de fluido vaporizado sobre a 
superfície interna dos tubos.

O balanço de energia mecânica é composto de três termos: cinético; gravitacional, e um termo devido ao atrito. Assim, pode-se escrever, para escoamento monofásico:

$-\frac{d P}{d L}=-\frac{G^{2}}{\rho^{2}} \frac{d \rho}{d L}+\rho g \operatorname{sen} \theta+\frac{f G^{2}}{2 D \rho}$

Já para o escoamento bifásico, a equação necessita de algumas modificações:

$-\frac{d P}{d L}=-\frac{G^{2}}{\rho_{t p}^{2}} \frac{d \rho_{t p}}{d L}+\rho_{t p} g \operatorname{sen} \theta+\varphi_{l o}^{2} \frac{f G^{2}}{2 D \rho}$

$\mathrm{Na}$ Equação 9, acrescentou-se a massa específica bifásica $\left(\rho_{t p}\right)$ e o coeficiente $\left(\varphi_{l o}^{2}\right)$ (Smith, 1986). A massa específica bifásica é calculada em função das massas específicas do líquido e do gás. Já o coeficiente $\varphi_{l o}^{2}$, é calculado para adequar o termo referente ao atrito monofásico para o bifásico.

A equação utilizada para o balanço de massa foi:

$m T=m V+m L$

A taxa de entalpia é uma função de estado e, portanto, o valor desta propriedade para uma dada substância dependerá, além da temperatura e pressão, do estado de agregação em que se encontra esta substância.

$H=m L C p l(T-T o)+m V C p l(T s a t-T o)+m V \lambda$

Para estimar pressão de vapor em função da temperatura do sistema ou a temperatura de saturação em função da pressão do sistema, foi utilizada a Equação de Antoine. Além disso, foram necessárias outras equações empíricas, descritas a seguir.

O fator de atrito monofásico foi calculado pela equação de Serghides (1984).

$f=\left[A-\frac{(B-A)^{2}}{(C-2 B+A)}\right]^{-2}$

sendo:

$$
\begin{aligned}
& A=-2 \log \left[\left(\frac{\varepsilon}{3,7 D}\right)+\left(\frac{12}{R e}\right)\right] \\
& B=-2 \log \left[\left(\frac{\varepsilon}{3,7 D}\right)+\left(\frac{2,51 A}{R e}\right)\right] \\
& C=-2 \log \left[\left(\frac{\varepsilon}{3,7 D}\right)+\left(\frac{2,51 B}{R e}\right)\right]
\end{aligned}
$$

A massa específica do líquido foi calculada pela Equação de Rackett (Spencer e Danner, 1972) e a do gás pela equação de gás ideal. 
A viscosidade do líquido foi calculada pela equação de Guzman-Andrade (Poling et al, 2001).

$\mu_{l}\left(\frac{k g}{m s}\right)=A e^{B / T}\left({ }^{\circ} C\right)$

Já a do gás foi calculada por um polinômio, obtido por regressão linear da viscosidade em função da temperatura, de acordo com Spiers (1977), para a água:

$\mu_{g}\left(\frac{k g}{m s}\right)=-0,419910^{-5}+0,4533610^{-7} T-0,5074910^{-11} T^{2}+0,1552610^{-14} T^{3}(K)$

As capacidades caloríficas do líquido e do gás foram calculadas de acordo com Smith et al. (2007):

$\frac{C p_{l}}{R}=8,712+1,2510^{-3} T-0,1810^{-6}(K)$

$\frac{C p_{g}}{R}=3.470+1,45010^{-3} T+0,12110^{5} T^{-2}(K)$

Para a condutividade térmica do líquido, para o calor de vaporização e para a tensão superficial, utilizaram-se equações obtidas por regressão linear a partir de dados retirados de Incropera et al. (2008):

$k_{l}\left(\frac{W}{m K}\right)=0,0011 T+0,2745(K)$

$\lambda\left(\frac{k J}{k g}\right)=-2,4324 T+3167,2(K)$

$\sigma\left(\frac{N}{m}\right)=-0,0002 T+0,1223(K)$

\section{SIMULAÇÃO}

As rotinas de simulação do refervedor da torre de destilação foram implementadas no software Scilab, ambiente de programação livre muito similar ao Matlab. O problema foi dividido em quatro regiões, conforme mostra a Figura 1.

A Região 1 compreende a saída de líquido da torre de destilação, até a entrada do trocador de calor. Nessa região, as variações ocorrem somente em função da perda de carga ao longo do trajeto, com isso somente a Equação 8 foi utilizada. A Região 2 foi designada como a entrada do trocador até o momento em que a temperatura do sistema $(T)$ se iguala à temperatura de saturação (Tsat), como pode ser visto na Figura 5, momento esse que se inicia a vaporização. A determinação desse ponto, no qual Tsat se iguala a $T$, foi realizada pelo método iterativo da Bisseção (Lage e Pinto, 2001) conforme esquema representado na Figura 2. Nessa região, utilizaram-se as Equações 6, 8, 10,11 , com $m V=0$. 


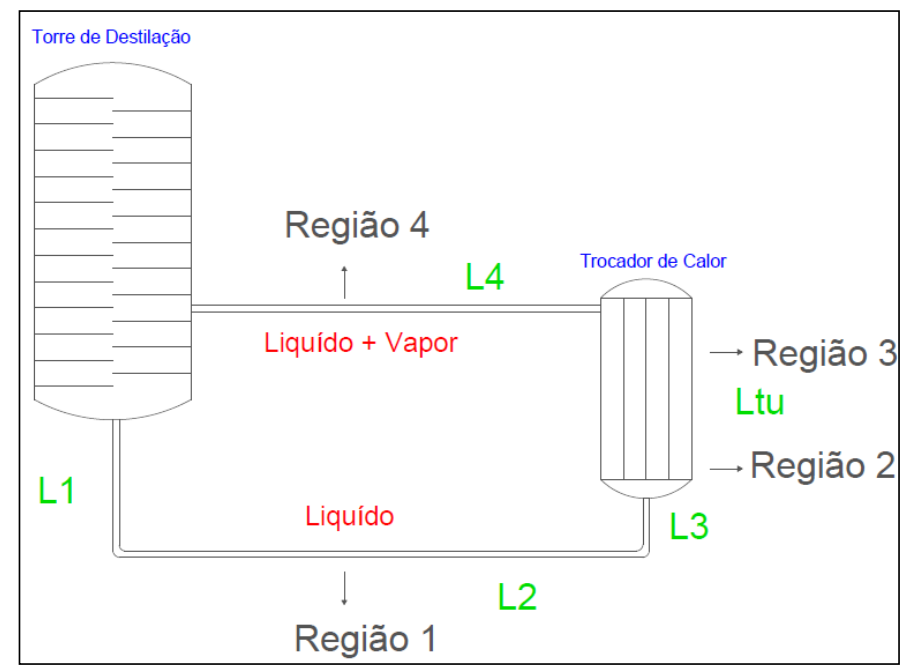

Figura 1 - Representação esquemática.

A Região 3 inicia-se com a vaporização e compreende o restante do trocador de calor. Nessa região, utilizaram-se as Equações 6, 9, 10 e 11, além da equação de Antoine. Já a Região 4 é compreendida pelo retorno para a torre de destilação da mistura bifásica da saída do trocador de calor. As Equações utilizadas foram: 9, 10 e 11, além da equação de Antoine.

Assim, tem-se um sistema de equações algébrico-diferencial (índice diferencial igual a 1) que é resolvido através da rotina DASSL (Petzold, 1983). O problema exige ainda uma convergência externa, uma vez que a pressão inicial do circuito tem que ser igual à pressão final (ambos os pontos representam o fundo da torre). Neste caso, o método iterativo utilizado foi a o método da secante (Chapra e Canale, 2011). A representação esquemática pode ser vista na Figura 3.

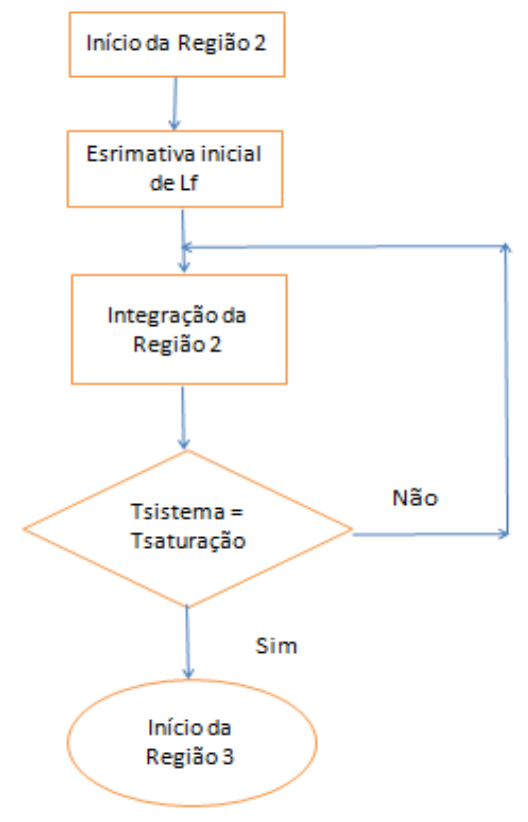

Figura 2 - Determinação do ponto de início da Vaporização $(L f)$.

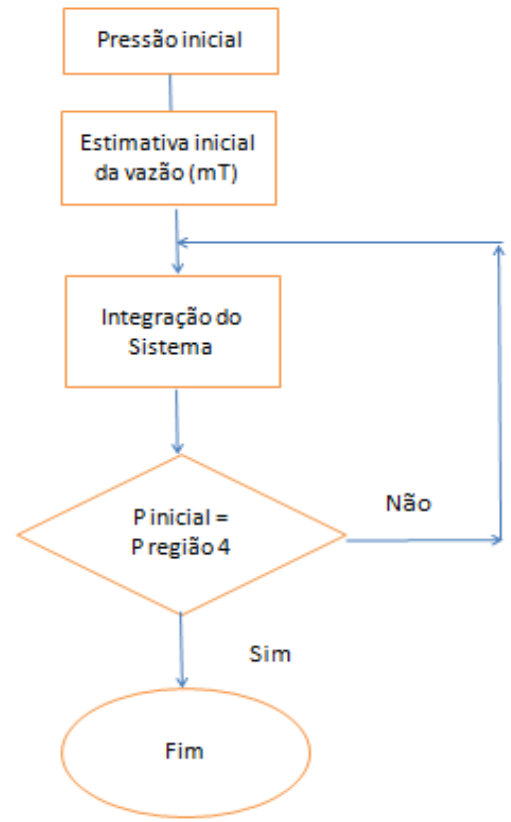

Figura 3 - Determinação da vazão do sistema $(m T)$. 


\section{RESULTADOS NUMÉRICOS}

Para exemplificar a utilização dos algoritmos de simulação desenvolvidos, criou-se um exemplo referente à vaporização de uma substância pura (água). Na Tabela 1, encontramos o serviço térmico e na Tabela 2 o exemplo do trocador de calor.

Tabela 1- Dados das correntes

\begin{tabular}{cc}
\hline Corrente de processo & Água \\
Temperatura de entrada $(\mathrm{K})$ & 373 \\
Pressão de entrada $(\mathrm{Pa})$ & 101325 \\
Resistência de depósito $\left(\mathrm{m}^{2} \mathrm{~K} / \mathrm{W}\right)$ & 0,00053 \\
Utilidade & Vapor saturado \\
Temperatura de entrada $(\mathrm{K})$ & 415 \\
Resistência de depósito $\left(\mathrm{m}^{2} \mathrm{~K} / \mathrm{W}\right)$ & 0,000088 \\
\hline & \\
Tabela 2- Dados mecânicos dos trocadores \\
\hline Área (m²) & 18 \\
Comprimento dos tubos (m) & 1,5 \\
Número de tubos & 200 \\
Diâmetro interno dos tubos (m) & 0,01575 \\
Diâmetro externo dos tubos (m) & 0,01905 \\
\hline
\end{tabular}

A Figura 4 apresenta os perfis de pressão, temperatura, vazão de vapor e vazão líquido ao longo do sistema. Nesta figura, é possível perceber vários comportamento típicos de termossifões: (i) a pressão inicialmente aumenta em função da coluna de líquido em escoamento descendente e depois diminui no escoamento, inicialmente monofásico e depois bifásico, no sentido oposto, (ii) a temperatura aumenta no trocador até atingir a saturação e depois diminui em função da queda de pressão, (iii) a fração vaporizada aumenta no interior do trocador em função da taxa de transferência de calor. Particularmente, as variações da temperatura do sistema e da temperatura de saturação podem ser visualizadas na Figura 5, evidenciando o momento no qual se inicia a vaporização (ou seja, a transição da região 2 para a região 3 ).

Avaliando o comportamento global do sistema, a fração vaporizada alcança 9,09\%, que está de acordo com os limites especificados para água e soluções aquosas que são de $2 \%$ até $10 \%$ (Palen, 2008).

\section{CONCLUSÕES}

O presente trabalho apresenta um algoritmo de simulação capaz de prever o comportamento de um termossifão vertical. Em função da natureza do equipamento, a análise não se baseia apenas em equações integradas na forma analítica, como é comum em estudos envolvendo trocadores de calor (método LMTD e da efetividade), porém implica na modelagem simultânea de balanços de massa, momento e energia, resultando em um sistema de equações algébrico-diferenciais. 

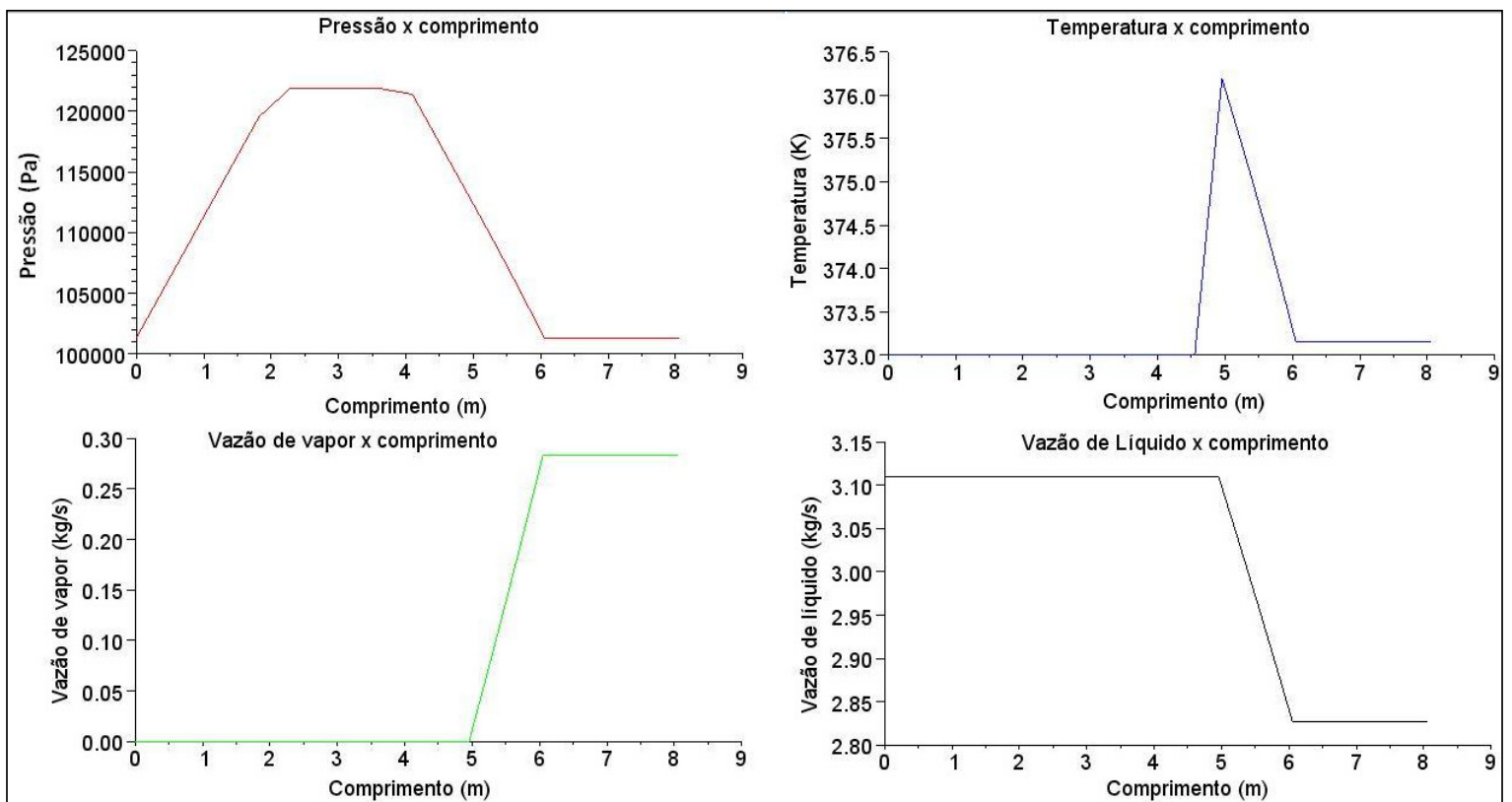

Figura 4 - Perfis de pressão, temperatura, vazão de vapor e vazão de líquido.

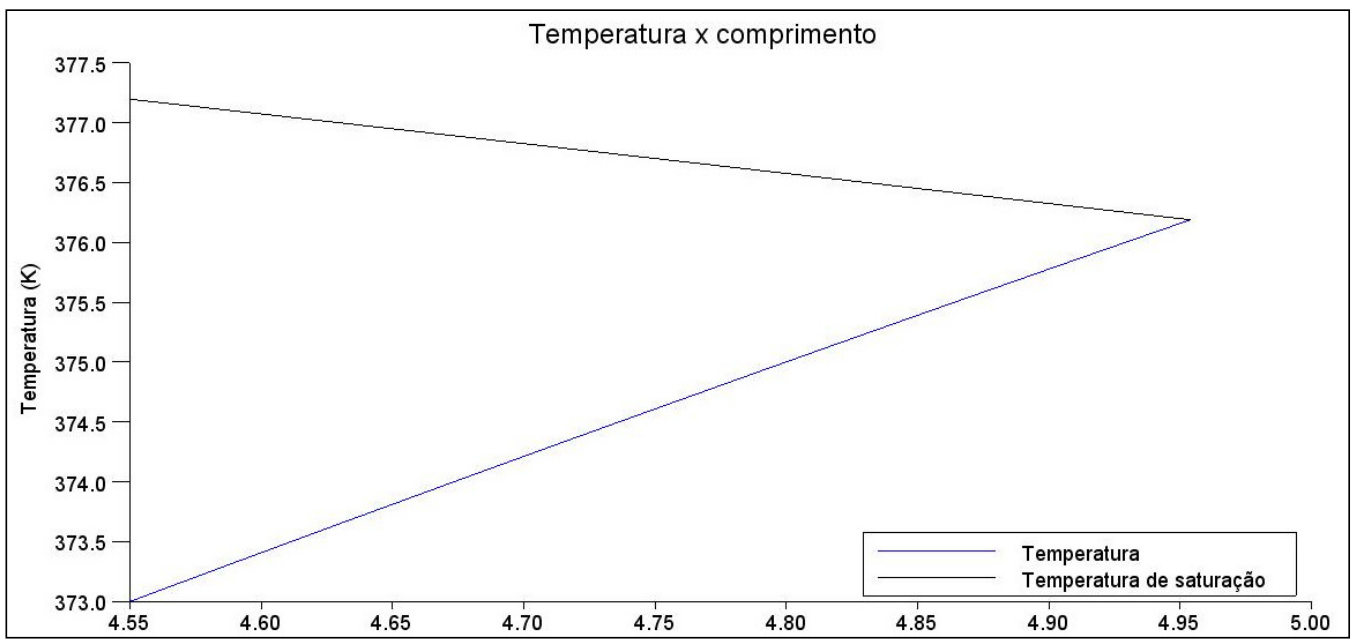

Figura 5 - Perfis temperatura de saturação e temperatura para região 2.

\section{NOMENCLATURA}

A área de troca térmica, $\mathrm{m}^{2}$

$\mathrm{Cpl}$ capacidade calorífica do liquido, $\mathrm{J} /(\mathrm{kg} \mathrm{K})$

Cpg capacidade calorífica do gás, $\mathrm{J} /(\mathrm{kg}$

$\mathrm{K})$

D diâmetro, $\mathrm{m}$

Dte diâmetro externo dos tubos, $\mathrm{m}$

Dti diâmetro interno dos tubos, $\mathrm{m}$ $f \quad$ fator de atrito

$g$ aceleração da gravidade, $\mathrm{m} / \mathrm{s}^{2}$

$G \quad$ fluxo mássico, $\mathrm{kg} /\left(\mathrm{m}^{2} \mathrm{~s}\right)$

$H \quad$ taxa de entalpia, $\mathrm{J} / \mathrm{s}$

$k w$ condutividade térmica da parede, $\mathrm{W} / \mathrm{m} \mathrm{K}$

$L \quad$ comprimento, $\mathrm{m}$

$m L$ vazão mássica de liquido, $\mathrm{kg} / \mathrm{s}$ 
$m T \quad$ vazão mássica total, $\mathrm{kg} / \mathrm{s}$

$m V \quad$ vazão mássica de vapor, $\mathrm{kg} / \mathrm{s}$

$N t$ número total de tubos

$P$ pressão, $\mathrm{Pa}$

$Q \quad$ taxa de transferência de calor, $\mathrm{J} / \mathrm{s}$

$R$ constante universal dos gases,

$\mathrm{J} /(\mathrm{mol} \mathrm{K})$

Rfe resistência de depósito externa, $\mathrm{m}^{2} \mathrm{~K} / \mathrm{W}$

$R f i \quad$ resistência de depósito interna, $\mathrm{m}^{2}$ $\mathrm{K} / \mathrm{W}$

$T \quad$ temperatura, $\mathrm{K}$

$T_{H} \quad$ temperatura da corrente quente, $\mathrm{K}$
To temperatura de referência, $\mathrm{K}$

Tsat temperatura de saturação, $\mathrm{K}$

$U$ coeficiente global de transferência

de calor, $\mathrm{W} /\left(\mathrm{m}^{2} \mathrm{~K}\right)$

$v \quad$ velocidade de escoamento, $\mathrm{m} / \mathrm{s}$

$\theta$ ângulo

$\rho \quad$ massa específica, $\mathrm{kg} / \mathrm{m}^{3}$

$\rho_{t p} \quad$ massa específica da mistura bifásica, $\mathrm{kg} / \mathrm{m}^{3}$

$\varphi_{l o}{ }^{2} \quad$ coeficiente calculado para adequar o termo referente ao atrito monofásico para o bifásico

\section{REFERÊNCIAS}

CALDAS, J.N. (Organizador)...[et al]. Internos de Torres: pratos e recheio. 2. ed. Rio de Janeiro: Interciência: PETROBRAS, 2007.

CHAPRA, S.C.; CANALE, R.P. Métodos numéricos para engenharia. 5. ed. Editora AMGH, Porto Alegre, 2011.

INCROPERA, F.P.;DEWITT, D.P.; BERGMAN, T. L.; LAVINE, A.S. Fundamentos de Transferência de Calor e Massa. Rio de Janeiro: LTC- Livros Técnicos e Científicos Editora S.A., 2008.

INDIO DO BRASIL, N. Introdução à engenharia química. 2. ed. Rio de Janeiro: Interciência: PETROBRAS, 2004.

LAGE, P.L.C.; PINTO, J.C.C.S. Métodos Numéricos em Problemas de Engenharia Química.

Rio de Janeiro: e-papers, 2001.

PALEN, J. W., Shell-and-Tube Reboilers. In: Heat Exchanger Design Handbook, Ed. G. F. Hewitt, Begell House, 2008.

PETZOLD, L.R. A Descriptions of DASSL: A differential/algebraic system solver, in: Scientific Computing, R.S. Stepleman et al., eds., 65-68, North-Holland, Amsterdam, 1983.

POLING, B.E.; PRAUSNITZ, M.; O'CONNELL, J.P. The Properties of Gases and Liquids, 5th ed. McGRAW-HILL, 2001.

SERGHIDES, T.K. Estimate friction factor accurately. Chem. Eng., 91(5): 63-64. 1984.

SMITH, J.M.; VAN NESS, H.C.; ABBOTT, M.M. Introdução à Termodinâmica da Engenharia Química. 7. Ed. Rio de Janeiro: Editora LTC, 2007.

SMITH, R.A. Vaporisers: selection, design and operation. Longman Scientific and Technical, UK, 1986.

SPENCER, C.F.; DANNER, R.P. Improved Equation for Prediction of Saturated liquid Density. Journal of Chemical and Engineering Data, vol 17, No.2, 1972.

SPIERS, H.M. Technical Data on Fuel, 7th ed. British National Commitee, World Energy Conference, London, 1977. 